Publ. Mat. 59 (2015), 45-54

DOI: 10.5565 /PUBLMAT_59115_03

\title{
BLASCHKE PRODUCTS AND NEVANLINNA-PICK INTERPOLATION
}

\author{
ARNE STRAY
}

\begin{abstract}
For a Nevanlinna-Pick problem with more than one solution, Rolf Nevanlinna proved that all extremal solutions are inner functions. If the interpolation points are contained in finitely many cones terminating at the unit circle, it is shown that all extremal solutions are Blaschke products.
\end{abstract}

2010 Mathematics Subject Classification: 30E05; Secondary: 30J10.

Key words: Blaschke product, minimal interpolation, logarithmic capacity.

Let $U$ denote the set of all analytic functions $f$ bounded by one in modulus in the unit disc $D=\{z:|z|<1\}$. We shall assume there are at least two different solutions to the Nevanlinna-Pick interpolation problem

$$
f\left(z_{n}\right)=w_{n}, \quad n=1,2, \ldots, f \in U .
$$

If this happens, the problem is called indeterminate. Let $E$ denote the set of all solutions to $(*)$. R. Nevanlinna found functions $P, Q, R$, and $S$, analytic in $D$, such that $E$ is parametrized by $U$ in the following way:

$$
E=\left\{\frac{P-Q w}{R-S w}, w \in U\right\} .
$$

It is part of Nevanlinna's discovery that for $w \equiv e^{i \alpha}, 0 \leq \alpha<2 \pi$, the corresponding solution $I_{\alpha}=\frac{P-Q e^{i \alpha}}{R-S e^{i \alpha}}$ is an inner function. It is a consequence of Nevanlinna's formula that the "Wertevorrat"

$$
\triangle(z)=\{f(z): f \in U, f \text { solves }(*)\}
$$

is a disc and that the boundary points of $\triangle(z)$ is in one-one correspondence with

$$
\left\{I_{\alpha}(z), 0 \leq \alpha<2 \pi\right\} .
$$

For this reason the set $\left\{I_{\alpha}, 0 \leq \alpha<2 \pi\right\}$ are often called the extremal solutions of $(*)$. In [5] it was proved that for almost all $\alpha, I_{\alpha}$ is a Blaschke product, where the exceptional set of $\alpha$-values has zero logarithmic capacity. If the interpolation problem (*) involves only a finite 
number of points, it is a classical fact that all $I_{\alpha}$ are Blaschke products. A similar conclusion was obtained in [4] in the rather special situation where $S=\left\{z_{n}\right\}$ is an interpolating sequence for the bounded analytic functions in $D$ and where limsup $\left|w_{n}\right|$ is sufficiently small. The present work complements the results in [4] and [5] as follows. First we find a rather precise condition on the sequence $S=\left\{z_{n}\right\}$ giving that for any indeterminate problem $(*)$ all $I_{\alpha}$ are Blaschke products. Next we show that for any closed set $K$ of zero logarithmic capacity on the unit circle $T$, there is a Nevanlinna-Pick problem $(*)$ such that $I_{\alpha}$ is a Blaschke product if and only if $e^{i \alpha} \in T \backslash K$.

Theorem 1. Given a Nevanlinna-Pick interpolation problem $f\left(z_{n}\right)=$ $w_{n}, n=1,2, \ldots, f \in U$ with more than one solution, if $S=\left\{z_{n}\right\}$ is contained in a finite number of Stolz angles, then any extremal solution is a Blaschke product.

As mentioned above, for an arbitrary indeterminate Nevanlinna-Pick problem, almost all extremal solutions are Blaschke products, the exceptional set of $\alpha$-values having zero logarithmic capacity. It seems that for sequences $S=\left\{z_{n}\right\}$ clustering on a small subset of the unit circle, the exceptional set of $\alpha$-values should be described by some finer measure than logarithmic capacity. As an example we mention:

Theorem 2. Given an indeterminate Nevanlinna-Pick interpolation problem $f\left(z_{n}\right)=w_{n}, n=1,2, \ldots, f \in U$, if $S=\left\{z_{n}\right\}$ has $M$ limit points on the unit circle, then at most $M$ of the extremal solutions fail to be a Blaschke product.

Finally we complement Theorem 1 and Theorem 2 with the following:

Theorem 3. Let $K$ denote a compact subset of the unit circle having zero logarithmic capacity. Then there is a Nevanlinna-Pick problem where $I_{\alpha}$ is a singular inner function if and only if $e^{i \alpha} \in K$ and an interpolating Blaschke product for other values of $e^{i \alpha}$.

It seems that Theorem 3 should be true for more general sets $K$ having zero logarithmic capacity.

Proof of Theorem 1: Let $\Gamma$ denote the points in the unit disc inside the cone given by

$$
\{z:-\phi<\arg (1-z)<\phi\},
$$

where $\phi$ is some fixed angle, $0<\phi<\frac{\pi}{2}$. Let $\phi<\psi<\frac{\pi}{2}$ and let $\widetilde{\Gamma}$ denote the cone corresponding to $\psi$. 
We assume the interpolation points $z_{n}$ are contained in $\Gamma$ and a finite number of rotated copies of $\Gamma$. We also consider $\widetilde{\Gamma}$ and its corresponding rotations.

Let us assume that some extremal solution $J$ of $(*)$ contains a singular inner function

$$
I(z)=\exp \left(-t \frac{1+z}{1-z}\right)
$$

as a factor, where $t$ is a positive constant. Using Nevanlinna's parametrization formula, we shall obtain a contradiction.

We fix a positive integer $N$ and let

$$
\Gamma_{N}=\left\{z: z \in \Gamma, 0<1-|z|<2^{-N}\right\}
$$

and

$$
\widetilde{\Gamma_{N}}=\left\{z: z \in \widetilde{\Gamma}, 0<1-|z|<2^{-N+1}\right\} .
$$

Let $\Pi(z)$ denote the Blaschke product

$$
\Pi(z)=\prod \frac{\left|z_{n}\right|}{z_{n}} \frac{z_{n}-z}{1-\overline{z_{n}} z}
$$

and denote by $\Pi_{N}$ the subproduct consisting of the factors with its zero in $\Gamma_{N}$. Consider a point $z \in D$ such that the pseudohyperbolic distance

$$
\sigma\left(z, z_{n}\right)=\frac{\left|z-z_{n}\right|}{\left|1-\overline{z_{n}} z\right|}
$$

exceeds some positive constant $a$ for all $n$. Then it is well known (see [3, p. 279]) that

$$
\begin{aligned}
4 \sum \frac{(1-|z|)\left(1-\left|z_{n}\right|\right)}{\left|1-\overline{z_{n}} z\right|^{2}} & \leq \log |\Pi(z)|^{-2} \\
& \leq\left(1+2 \log \frac{1}{a}\right) 4 \sum \frac{(1-|z|)\left(1-\left|z_{n}\right|\right)}{\left|1-\overline{z_{n}} z\right|^{2}} .
\end{aligned}
$$

We apply the second inequality in (1) with $\Pi$ replaced by $\Pi_{N}$ and $z \in D$ located on $\partial \widetilde{\Gamma_{N}}$. The constant $a$ in (1) depend on $\phi$ and $\psi$, but nothing else.

Assume that $M_{k}$ is the number of zeros of $\Pi_{N}$ in $\left\{z: 2^{-k-1} \leq|z| \leq\right.$ $\left.2^{-k}\right\}$. Since

$$
\left|\frac{1}{1-\overline{z_{n}} z}\right|=\left|\frac{z-z_{n}}{1-\overline{z_{n}} z}\right|\left|\frac{1}{z-z_{n}}\right|
$$


and $z \in \partial \widetilde{\Gamma_{N}}$ we have

$$
\left|\frac{1}{z-z_{n}}\right| \leq \frac{C}{1-|z|}
$$

with $C$ depending only on $\phi$ and $\psi$. We conclude that

$$
\log \left|\Pi_{N}(z)\right|^{-2} \leq \frac{C}{1-|z|} \sum_{k=N}^{\infty} 2^{-k} M_{k}<\frac{\epsilon}{1-|z|}
$$

for given $\epsilon$ if $N$ is large. This gives us the lower bound

$$
\left|\Pi_{N}(z)\right| \geq e^{-\frac{\epsilon}{1-|z|}}
$$

on $\partial \widetilde{\Gamma_{N}}$. For the inner function $I$, assumed to be a factor in some extremal solution $J$ of $(*)$, we have for a given small number $\lambda$ that

$$
\left|\frac{I^{\frac{1}{2}}}{\Pi_{N}}\right|<\lambda
$$

on $\widetilde{\Gamma_{N}}$ provided $N$ is large, forcing $\epsilon$ to be as small as we wish.

In the following we consider the reduced interpolation problem

$$
(*)_{N} \quad f\left(z_{\nu}\right)=w_{\nu}, \quad \nu=1,2, \ldots,
$$

where $z_{\nu}$ denotes the zeros of $\Pi_{N}$. Let the solutions of $(*)_{N}$ be parametrized by the functions $P_{N}, Q_{N}, R_{N}$, and $S_{N}$.

Using the method of dual extremal problems as presented in the proof of Carleson lemma in [2], we can conclude that for given $\delta>0$, we can choose $\lambda$ so small that $(*)_{N}$ has a solution satisfying

$$
\|h\|_{\infty}<\delta
$$

and

$$
|h(z)| \leq \delta\left|\Pi_{N}(z)\right|
$$

uniformly on $\partial \widetilde{\Gamma_{N}}$. Indeed we find $h=g I^{\frac{1}{2}}$ where $g$ interpolates $J I^{\frac{-1}{2}}$ at the zeros of $\Pi_{N}$.

To obtain $g$, we just have to replace the Carleson contour $\Gamma$ in $[\mathbf{2}]$ by $\partial \widetilde{\Gamma_{N}}$ and observe that arc length restricted to $\partial \widetilde{\Gamma_{N}}$ is a Carleson measure.

Let us recall that for the general problem (*), the functions $P, Q$, $R$, and $S$ admit analytic continuation across the unit circle where $\left\{z_{n}\right\}$ doesn't cluster. Hence the same is true for the extremal solutions $I_{\alpha}$. We may also represent these extremal solutions as

$$
J_{w}=\frac{P_{N}-Q_{N} w}{R_{N}-S_{N} w},
$$


where $w$ has to be a solution to the Nevanlinna-Pick problem

$$
\frac{P_{N}\left(z_{\mu}\right)-Q_{N}\left(z_{\mu}\right) w\left(z_{\mu}\right)}{R_{N}\left(z_{\mu}\right)-S_{N}\left(z_{\mu}\right) w\left(z_{\mu}\right)}=w_{\mu},
$$

where $\left\{z_{\mu}\right\}$ represents the points from $\left\{z_{n}\right\}$ not being a zero of $\Pi_{N}$.

Since $(*)$ is indeterminate, so is also $(* *)$ and evidently $J_{u}$ is an extremal solution to $(*)$ if and only if $u$ is an extremal solution to $(* *)$. From (2) it follows that $R_{N}$ belongs to the Hardy space $H^{2}$ (see $[6]$ ). This will be shown to contradict certain lower bounds on $\left|R_{N}(z)\right|$ on certain subsets of $D$.

Now fix a point $z$ on $\partial \widetilde{\Gamma_{N}}$. The radius $\rho_{N}(z)$ of the Wertevorrat $\triangle_{N}(z)$ corresponding to $(*)_{N}$ satisfies

$$
(1-\delta)\left|\Pi_{N}(z)\right| \leq \rho_{N}(z) \leq\left|\Pi_{N}(z)\right|
$$

since the Wertevorrat contains all values of the form $h(z)+\zeta \Pi_{N}(z)$ with $|\zeta|<1-\delta$.

For $z \in \partial \widetilde{\Gamma_{N}}$ it follows from (2) and (3) that the Wertevorrat $\triangle_{N}(z)$ is nearly concentric around the origin. It follows from Schwarz lemma applied to the mapping

$$
w \rightarrow \frac{P_{N}-Q_{N} w}{R_{N}-S_{N} w}
$$

that

$$
\left|\frac{c(z)-J(z)}{\rho_{N}(z)}\right|=\sigma(u(z), \overline{a(z)}) \leq t<1,
$$

where $c(z)$ is the center of $\triangle_{N}(z)$ and $a(z)=\frac{S_{N}(z)}{R_{N}(z)}$. Here our extremal solution $J$ to $(*)$ is written as

$$
J=\frac{P_{N}-Q_{N} u}{R_{N}-S_{N} u}
$$

where $u$ is some extremal solution to $(* *)$. Since $u$ is inner and analytic near $z=1$, we have the estimate

$$
|u(z)| \geq 1-c(1-|z|)
$$

near 1. From (4) and (5) we deduce

$$
\left|\frac{S_{N}}{R_{N}}(z)\right| \geq 1-d(1-|z|),
$$

where $d$ depends only on $t$ in (4) and $c$ in (5). We then use our lower bound

$$
\rho_{N}(z)=\frac{\left|\Pi_{N}(z)\right|}{\left|R_{N}(z)\right|^{2}-\left|S_{N}(z)\right|^{2}} \geq(1-\delta)\left|\Pi_{N}(z)\right|
$$


to obtain the lower bound

$$
\left|R_{N}(z)\right|^{2} \geq \frac{\delta}{d(1-|z|)}
$$

uniformly on $\partial \widetilde{\Gamma_{N}}$. This is in contradiction with the Hardy-Littlewood maximal theorem since it is well known that $R_{N}$ belongs to the Hardy space $H^{2}$. The proof of Theorem 1 is complete.

Proof of Theorem 2: Given the fact that $P S-R Q=\Pi$, we find

$$
I_{\alpha}-I_{\beta}=\left(\left(e^{i \alpha}-e^{i \beta}\right) \Pi\right)\left(\left(R-S e^{i \alpha}\right)\left(R-S e^{i \beta}\right)\right)^{-1} .
$$

Since $\left(\left(R-S e^{i \alpha}\right)\left(R-S e^{i \beta}\right)\right)^{-1}$ is known to be an outer function and each $I_{\alpha}$ is analytic near the unit circle except for the limit points of $S$, this means that two different extremal solutions of $(*)$ cannot have a common singular factor corresponding to a point mass on the unit circle.

Proof of Theorem 3: Let $M$ be a compact subset of the open unit disc $D$ with zero logarithmic capacity, and consider a universal covering map

$$
\phi: D \rightarrow D \backslash M \text {. }
$$

It is well known that $\phi$ is an inner function. We shall assume that $0 \in M$ and from this it follows that $\phi$ must be a singular inner function. Let $u$ denote a point in $D \backslash M$ and define

$$
\phi_{u}=\frac{\phi-u}{1-\bar{u} \phi} .
$$

We claim that $\phi_{u}$ is a Blaschke product. To see this, consider a point $z_{0}$ such that $\phi\left(z_{0}\right)=u$. We can assume $z_{0}$ is an interior point of some fundamental domain $\Omega$ for the covering $\phi$. Since $\phi$ is univalent on $\Omega$, there is a small pseudohyperbolic disc $D_{0}$ around $z_{0}$ such that

$$
D_{0} \supset\{z:|\phi(z)-u|<\epsilon\} \cap \Omega
$$

provided $\epsilon$ is sufficiently small. If $\phi_{u}$ contained a singular inner factor, there would be a Stolz angle $\Gamma$ of opening close to $\pi$, such that $\phi(z) \rightarrow u$ uniformly inside $\Gamma$ as $|z| \rightarrow 1$ inside $\Gamma$. But then $\phi \approx u$ in large pseudohyperbolic discs inside $\Gamma$ and since $\phi$ is automorphic, this contradicts $(8)$.

Let $S=\left\{z_{\nu}\right\}=\phi^{-1}\{u\}$. Consider the Nevanlinna-Pick problem $(* * *) \quad f\left(z_{\nu}\right)=u, \quad \nu=1,2, \ldots, f \in U$.

It is now evident that any solution $f$ to $(* * *)$ will be of the form $\frac{u+w \phi_{u}}{1+\bar{u} w \phi_{u}}$, where $w$ is an arbitrary analytic function bounded by one in modulus in $D$. We also see that the extremal solutions $I_{\alpha}$ to $(* * *)$ 
corresponds to $w=e^{i \alpha}, 0 \leq \alpha<2 \pi$. In particular $\phi=I_{0}$. For these extremal solutions it is evident that $I_{\alpha}$ is a singular inner function if and only if

$$
\phi \neq \frac{u\left(1-e^{i \alpha}\right)}{1-|u|^{2} e^{i \alpha}}
$$

in the unit disc. Let $\left|\zeta_{0}\right|=1$. Consider the mapping

$$
\Lambda: \zeta \rightarrow \frac{u\left(1-\overline{\zeta_{0}} \zeta\right)}{1-|u|^{2} \zeta \overline{\zeta_{0}}}
$$

taking the unit circle onto the circle centered at $\frac{u}{1+|u|^{2}}$ and containing the origin. If $K$ is a compact subset of $\{\zeta:|\zeta|=1\}$ containing the point $\zeta_{0}$ and of zero logarithmic capacity, we shall assume in the following that $M=\Lambda(K)$. It follows that $I_{\alpha}$ is a singular inner function whenever $\alpha \in K$. If $\alpha \in T \backslash K$, it follows that $I_{\alpha}$ is a Blaschke product. This follows in the same way as we showed the $\phi_{u}$ is a Blaschke product whenever $u \in D \backslash M$. To complete the proof, we need only show that the zeros of $\frac{\phi-b}{1-\bar{b} \phi}$ form an interpolating sequence if $b \in D \backslash M$. The map $\phi$ is automorphic with respect to a group of cover transformations $T$ of $D$ and the zeros is just the complete orbit of one fixed zero. If $B$ is one of the Blaschke products under consideration, we have to verify Carleson's product condition at the zeros of $B$. This condition can be written as

$$
\left|B^{\prime}(z)\right|\left(1-|z|^{2}\right) \geq \delta>0,
$$

where $\delta$ doesn't depend on the zero considered. Since $\phi$ is automorphic one finds that

$$
\left|B^{\prime}(z)\right|\left(1-|z|^{2}\right)=\left|B^{\prime}(T z)\right|\left(1-|T z|^{2}\right)
$$

for $z \in D$ and any cover transformation $T$, and so Carleson's condition follows.

This completes the proof of Theorem 3.

There is a strong connection between the problem considered here and the work by J. R. Akeroyd, D. Khavinson, and H. S. Shapiro in [1]. We say that a sequence in $D$ converges onesidedly to a point on the unit circle if it converges and stays on one side of a line through the limitpoint dividing the disc into two parts. The result from [1] we have in mind is the following: Let $B$ be a Blaschke product whose zero set is a finite union of sequences converging onesidedly. The *-invariant subspace $H^{2} \ominus B H^{2}$ of $H^{2}$ contains no singular inner functions.

To relate this result to our work, we shall call a Nevanlinna-Pick problem scaled if it can be solved by a function in $H^{\infty}$ of norm strictly 
less than one. The problem is called semiscaled if after excluding finitely many interpolation points, the reduced problem is scaled.

We then have

Theorem 4. Let $S=\left\{z_{n}\right\}$ be a finite union of Blaschke sequences $S_{i}$, $1 \leq i \leq m$ converging onesidedly to $m$ distinct points $\zeta_{i}$ on the unit circle. Suppose the reduced Nevanlinna-Pick problem corresponding to some $S_{i}$ is semiscaled. If the full problem has more than one solution, none of the extremal solutions can have a singular inner factor with a singularity at $\zeta_{i}$.

Let us first remark that Theorem 4 follows from the above cited result from [1] in the special case where the interpolation problem is scaled. The reason is that, for scaled problems, $P-Q \zeta \in H^{2} \ominus z B H^{2}$ for any constant $\zeta$. Indeed, integrating over the unit circle gives

$$
\int \overline{(P-Q \zeta)} z B h d \theta=\int(R \bar{\zeta}-S) z h d \theta=0
$$

for any $h \in H^{2}$. The formulas

$$
Q=-B \bar{R}, \quad P=-B \bar{S}
$$

valid on the unit circle $T$, dates back to Nevanlinna's original paper on the subject. These formulas also give that $P, Q, R$, and $S$ all belong to $H^{2} \ominus z B H^{2}$ in the scaled case. See $[\mathbf{3}]$ for details.

Let us prove Theorem 4: We assume $\zeta_{i}=1$ and consider the reduced scaled interpolation problem corresponding to $S_{i}$ minus a finite number of points from $S_{i}$. The set of all solutions to the reduced problem is parametrized by

$$
\frac{P_{r}-Q_{r} w}{R_{r}-S_{r} w} .
$$

Let $\Pi$ denote the Blaschke product corresponding to the reduced problem. We assume that the zeros $\left\{z_{n}\right\}$ of $\Pi$ are located above some nonvertical line trough 1 . Consider the half circle $C_{-}$in the lower half plane defined by

$$
1-|z|^{2}=|1-z|^{2} .
$$

Using equation (1) above it follows easily that for $z \in C_{-}$

$$
\lim _{z \rightarrow 1}|\Pi(z)|=1 \text {. }
$$

Using Theorem 1 in [6], it follows that the radius $\rho_{r}(z)$ of the corresponding Wertevorrat also tends to 1 along $C_{-}$. Hence the center $c_{z}$ of 
the Wertevorrat tends to 0 at the same time. Any extremal solution to the full problem in Theorem 4 will have the form

$$
J=\frac{P_{r}-Q_{r} I}{R_{r}-S_{r} I},
$$

where $I$ is an inner function analytic near $z=1$. If $J$ contains a singular inner factor $\exp \left(-t \frac{1+z}{1-z}\right)$ we conclude that the pseudohyperbolic distance

$$
\sigma\left(I(z), \overline{\left(\frac{S_{r}(z)}{R_{r}(z)}\right)}\right)=\frac{\left|c_{z}-J(z)\right|}{\rho_{r}(z)}
$$

is strictly less than one as $z \rightarrow 1$ along $C_{-}$. But then we arrive at a contradiction like in the proof of Theorem 1 , since the $H^{2}$-function $R_{r}$ must satisfy

$$
\left|R_{r}(z)\right|^{2} \geq d(1-|z|)^{-1}
$$

uniformly on $C_{-}$near $z=1$.

Final remarks. It is not clear if Theorem 4 holds without assuming there is a semiscaled reduced problem. A solution seems to depend on more detailed information about the subharmonic function $\left(|R|^{2}-|S|^{2}\right)^{-1}$.

Let us also mention two consequenses of Theorem 1. It follows immediately that the extremal Blaschke products in that theorem are all indestructible. Another consequence is that the function

$$
I(z)=\exp \left(-\frac{1+z}{1-z}\right)
$$

can never be a minimal solution to any Nevanlinna-Pick problem in a Stoltz angle terminating at $z=1$.

\section{References}

[1] J. R. Akeroyd, D. Khavinson, and H. S. Shapiro, Weak compactness in certain star-shift invariant subspaces, J. Funct. Anal. 202(1) (2003), 98-122. DOI: 10.1016/S0022-1236(03)00060-0.

[2] P. L. Duren, "Theory of $H^{p}$ spaces", Pure and Applied Mathematics 38, Academic Press, New York-London, 1970.

[3] J. B. GARNETT, "Bounded analytic functions", Revised first edition, Graduate Texts in Mathematics 236, Springer, New York, 2007.

[4] A. Stray, Minimal interpolation by Blaschke products, J. London Math. Soc. (2) 32(3) (1985), 488-496. DOI: $10.1112 / \mathrm{jlms} / \mathrm{s} 2-32$. 3.488 . 
[5] A. Stray, Minimal interpolation by Blaschke products. II, Bull. London Math. Soc. 20(4) (1988), 329-332. DOI: 10.1112/blms/20. 4.329 .

[6] A. Stray, Interpolating sequences and the Nevanlinna Pick problem, Publ. Mat. 35(2) (1991), 507-516. DOI: 10.5565/PUBLMAT $35291-14$.

Department of Mathematics

University of Bergen

5020 Bergen

Norway

E-mail address: Arne.Stray@math.uib.no

Primera versió rebuda el 17 de setembre de 2013,

darrera versió rebuda el 10 de desembre de 2013. 